were sacrificed half hour, one hour, two hours, four hours, eighteen hours and twenty four hours after the operation. Some of them were alived for 2 days to 20 days after the operation. We examined these groups of cerebral lesions and cerebral blood vessels with the histological method. Thirty minutes after the operation, the cerebral vascular lesions and cerebral lesions were not found by the ordinal histological methods.

One to two hours after the operation, the section revealed partially hemorrhage via vascular wall into the perivascular spaces but there were no vascular changes.

Four hours after the injection, the section showed the perivascular transudation of blood cells, the exsudation of serum and indian ink, however the angionecrosis could not be found.

Eighteen hours after the injection the section revealed the remarkable appearance of fibrin in perivascular spaces, there were also hemorrhage into the perivascular spaces, extending to the local cerebral tissue. Angionecrosis were found in some of the sections.

Twenty four hours after the operation the perivascular hemorrhage and white infarction were frequently found. There were proliferation of glia cells at the peripheral areas of the cerebral lesions and angionecrosis was common in the hemorrhagic infarction.

Two days after the operation, the section showed the new formation of blood vessels, the proliferation of glia cells and the appearance of fat granulated cells.

According to the changes of the above described, at the first the increased permeability of blood vessel could be occurred, the second hemorrhage could be followed, and then fibrin could be appear in cerebral vascular wall producing angionecrosis.

\title{
29. Anterior Cerebral Artery, Thrombosis and Hypoplasia of its Pars Circularis
}

\author{
Soji SuzuKI \\ Dept. of Radiology, Tokyo Medical \& Dental University \\ School of Medicine
}

It is well-known that the circle of Willis has various anomalies in its anatomical configuration and the so-called "textbook" circle of Willis is encountered in only approximately fifty per cent of the normal population. The string-like Pars Circularis of anterior cerebral artery is rather uncommon among the anomalies but should be always born in mind, because the overlook of the anomaly may lead 
to the tragic outcome in ligation of the carotid artery for intracranial aneurysms.

It is also important to know that thrombotic occlusion of the proximal portion of anterior cerebral artery is not always easy to be differentiated from the hypoplasia or aplasia of Pars Circularis.

I recently experienced two patients who showed unilateral absence of Pars Circularis of anterior cerebral artery on cerebral arteriograms.

The one was a 34-year-old female who had cancer of right maxilla with invasion of cancer to the base of the skull. Cerebral angiography revealed absent Pars Circularis of right anterior cerebral artery and later the autopsy disclosed thrombotic occlusion. The other was a 62-year-old male with lung cancer who developed a questionable evidence of metastasis to brain. Cerebral angiography revealed absent Pars Circularis of left anterior cerebral artery and the autopsy disclosed congenital hypoplasia of the portion.

\title{
30. Study on the Patient Suffering from the Closure of Arteria Cerebri Anterior
}

\author{
Hajime Teraoka, Kōichí Miura and Michio Yamagata \\ Neuro-psychiatric Department, Kumamoto University School of Medicine
}

Patient is 38 years old farmer. About 28 years old, he had focal epileptic convulsive fits, and has suffered from the disturbance of left lower extremity next to upper extremity since about 36 years old. hospital.

And he has felt stiff in his shoulders and bazzing in his ears and entered

Neurological main symptoms are following:

1) disturbance of motility of left extremity 2) rigidity 3) grasp-reflex and partial resistence 4) no disturbance of sensibility. mind.

It is worthy of note that these neurologic symptoms are apt to be influenced by

Psychiatric symptoms are following: dullness.

1) slight disturbances of perceptivity and memory. 2) euphoria 3) inental

At the right parietal part abnormal wave was found in electroencephalogram.

It is most worthy of note that the closure of right arteria cerebri anterior was found in angiography.

And the pulsation of the right arteria carotis communis is weakend. 